\title{
Ambient intelligence and ergonomics in Asia
}

\author{
Toly Chen · Chu-Chai Chan • \\ Hsin-Chieh Wu $\cdot$ Yu-Cheng Lin
}

Received: 21 October 2014 / Accepted: 24 October 2014/Published online: 8 November 2014

(C) Springer-Verlag Berlin Heidelberg 2014

Ambient intelligence $(\mathrm{AmI})$ is one of the most important advances in computer technology in the mobile era. By being aware and applying knowledge of ergonomics (human factors), AmI system users will be in better shape and health, become more effective and find using AmI system a more pleasant and enjoyable experience.

This special issue contains selected papers from the 1 st international conference on ambient intelligence and ergonomics in Asia (AmIE 2013), which was held at Taichung city, Taiwan, July 3-5, 2013. The conference attracted a large number of scientific papers that contributed to the state-of-the-art in the fields of ambient intelligence and ergonomics. After a strict review, five articles from researchers around the world were finally accepted.

To determine an understandable algorithm that could be used by designers to create novel concept designs, M.T. Wang and C.C. Yang selected the popular motor scooter as a sample product, and used the most distinctive front handle cover as a design target. Their method included three phases: preparation, construction of conceptual creativity, and semantic analysis. After comparing random idea sketches with designs available on the market, they observed that certain concept designs obtained using this

T. Chen $(\bowtie)$

Department of Industrial Engineering and Systems Management, Feng Chia University, Taichung, Taiwan

e-mail: tcchen@fcu.edu.tw

C.-C. Chan - H.-C. Wu

Department of Industrial Engineering and Management, Chaoyang University of Technology, Taichung, Taiwan

Y.-C. Lin

Department of Industrial Engineering and Management, Overseas Chinese University, Xitun, Taiwan method were exceptionally innovative, and could be easily redesigned for an actual product.

A.M. Otebolaku and M.T. Andrade investigated context-aware recommendation techniques for implicit delivery of contextually relevant online media items. The proposed recommendation service identifies a user's dynamic contextual situation from the device's built-in sensors, and uses case-based reasoning to determine the user's current contextual preferences. The effectiveness of the proposed recommendation service was evaluated with a case study.

Gripping and pinching are frequently used hand strength in various occupational activities and in clinical evaluation of the hand. Therefore, formulating grip and pinch prediction models with easily obtainable personal parameters will help facilitate the design and evaluation of workplace environments or facilitate the hand impairment or progression assessments. P.C. Sung, C.C. Hsu, C.L. Lee, Y.-S.P. Chiu, and H.L. Chen developed maximum voluntary contraction (MVC) grip and key pinch strength prediction models using regression method and artificial neural networks (ANN).

An ANN is a prevalent humanized computing method that imitates the central nervous system of a human. To estimate the cycle time of a job in a wafer fabrication factory, H.C. Wu and T. Chen proposed a joint use of a classification and regression tree (CART) and back propagation network (BPN). In their method, a BPN is constructed to estimate the cycle times of jobs of a branch. A real case was used to evaluate the effectiveness of the proposed methodology.

C.T. Tseng, Y.L. Lee, and C.C. Chou designed an AmIbased decision support system that combined an electromagnetism-like mechanism (EM) and sensory data to aid human operators in making decisions regarding the 
management of cascade hydropower systems. The AmIbased system proposed in their study was used to determine the periodic water release at each plant in the Dajia hydropower system, thereby maximizing the Taiwan power company's power-generation profit.

We would like to thank to the JAIHC Editor-in-Chief Vincenzo Loia for providing full support in bringing out this special issue. We are thankful to the paper contributors who shared their research as well as the reviewers who spared their valuable time in paper review. We would also like to thank the journal staff. Without their support and professional assistance, the pre-publication process would not been possible. 